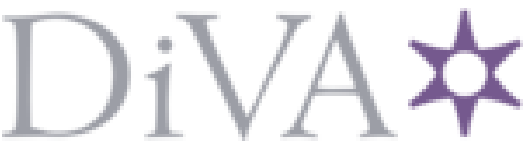

http://www.diva-portal.org

Postprint

This is the accepted version of a paper presented at The International Conference on Electrical Engineering (IECC2013), 14-17 July, 2013, Xiamen, China.

Citation for the original published paper:

Cheng, J., Robalino, D., Werelius, P., Ohlen, M. (2013)

Advanced Technique for Moisture Condition Assessment in Power Transformers

In: (pp. 718-723).

N.B. When citing this work, cite the original published paper.

Permanent link to this version:

http://urn.kb.se/resolve?urn=urn:nbn:se:kth:diva-30310o 


\title{
Advanced Technique for Moisture Condition Assessment in Power Transformers
}

\author{
JIALU CHENG ${ }^{1}$, DIEGO ROBALINO $^{2}$, PETER WERELIUS $^{3}$, MATZ OHLEN $^{3}$
}

\author{
${ }^{1}$ Megger China, ${ }^{2}$ Megger USA, ${ }^{3}$ Megger Sweden
}

\begin{abstract}
:
The presence of moisture in a transformer needs to be monitored throughout its service life. Moisture deteriorates transformer insulation by decreasing both electrical and mechanical strength. High moisture content accelerates solid insulation aging, reduces the breakdown strength and makes the transformer vulnerable to the overload conditions due to high temperature spot bubbling. In addition to that, partial discharge can occur in a high voltage region because of the moisture disturbance.

Traditional indirect estimation of the moisture concentration in the solid insulation of power transformers includes testing the oil samples as well as measurement of the insulation resistance and loss tangent $(50 / 60 \mathrm{~Hz})$ of the transformer. However, these methods usually give limited information and may lead to wrong conclusions. Direct measurement is not viable and may not be representative to take a paper sample from the surface insulation because moisture distribution is not homogeneous along the insulation geometry.

Dielectric Frequency Response, DFR was introduced more than 20 years ago and has been thoroughly evaluated and proven. Several documents have been published summarizing the research work and field tests all over the world. DFR is a practical non-intrusive and non-destructive technique for moisture condition assessment in power transformers, a breakthrough compared with traditional methods. Scientists, researchers and utility operators have shown great interest in the development and application of DFR technique.

In this paper, the limitation of traditional methods is presented at first. Later, a comprehensive review of DFR method demonstrates its advantages over traditional methods. Finally, latest research about the mathematical model, temperature correction and test voltage is included to answer common questions regarding the application of DFR method.
\end{abstract}

\section{Keywords:}

Moisture, power transformer, insulation resistance, dissipation factor, dielectric frequency response, recovery voltage, temperature correction

\section{Introduction}

Transformers are by far, one of the most mission critical components in the electrical grid. With the increasing amount of power transformers installed each year, the need for reliable diagnostic methods drives the world's leading experts to evaluate new technologies that improve reliability and optimize the use of the power network.

Moisture is one of the factors deteriorating the insulation of the power components. The presence of moisture in power transformers decreases both electrical and mechanical strength. Heat and moisture together put the insulation under accelerated aging. The aging of cellulose is greatly increased with the existence of moisture. It also reduces the breakdown strength of the power transformers and makes the transformers vulnerable to the overload conditions due to high temperature spot bubbling. The inception voltage of partial discharge also becomes lower with the presence of water [1]. Therefore, moisture inside power transformers must be periodically monitored throughout their service life in order to minimize the risk of unexpected failure.

A variety of methods are available for indirect estimation of moisture in the solid insulation, including: dielectric response in time domain and frequency domain as well as water content test of oil. These methods are widely used and correct understanding of limitations is required.

From all above listed techniques, dielectric response in the frequency domain has been proven to be an effective solution for power operators. DFR accurately determines the bulk moisture concentration in the solid insulation allowing manufacturers and operators to take assertive corrective or preventive actions that permit extension of the service life of power transformers.

\section{Review of the prevailing methods}

\subsection{Water content test in oil}

The insulation system of power transformers consists of oil and paper/cellulose. The moisture contents in paper/cellulose and in oil are related with each other. Researchers have established a correlation between moisture in oil vs. moisture in paper under the assumption of equilibrium between oil and paper at different temperatures named moisture equilibrium curves as shown in Figure 1 [1]. The figure indicates that if the water contained in oil is known, the moisture content of paper could be estimated based on the moisture equilibrium curves. Mineral oil from transformers is frequently sampled in field and afterwards the water content is measured by a reliable state of the art method - Karl Fischer Titration [2]. Then the moisture content of the solid insulation could be estimated.

ASTM D923-07 provides guidelines for sampling electrical insulating liquids. However, due to the low solubility of water in oil at room 
temperature (20 $\square \mathrm{C}$ ), factors like moisture ingress during oil sampling and transportation, different handling methods in the lab significantly reduce the reliability of the result. As for an example, tests on the same oil sample by seven laboratories across Europe revealed surprising differences as shown in Figure 2 [3]. None of them reasonably agrees with each other. To make things even worse, free water appears when the moisture is saturated in oil and deposits to the bottom which is not included in the oil samples.

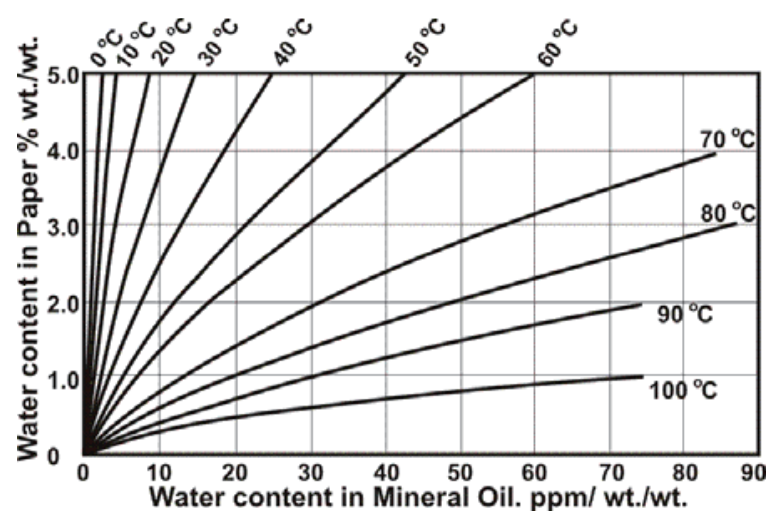

Figure 1 Water equilibrium curves in oil/paper system [1]

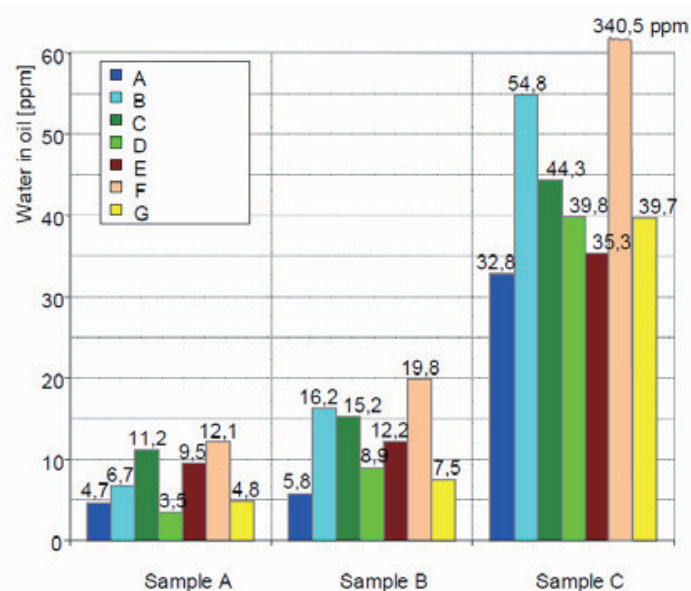

Figure 2 Moisture in oil in ppm measured by seven laboratories [3]

The solubility of water in oil is dependent on temperature. The moisture distribution in oil-paper system is usually not homogeneous along the insulation geometry of power transformers. All of these uncertainties impede this method to be a reliable estimation of moisture condition in transformers.

\subsection{Insulation resistance measurement}

The typical insulation resistance, Polarization Index or Dielectric Absorption Ratio tests have been regarded as useful tools in evaluating the condition of an insulation material. It is believed that if the insulation is contaminated with moisture or impurities, the polarization index will decrease. However, the successful application of the test method is limited to solid insulations such as motor insulation. Experiments have been carried out to prove the influence of mineral oil on the measured polarization index. The insulation resistance test on a transformer is made as usual. Then the aged mineral oil inside the transformer is drained out and replaced with new oil. The resistance curves are shown in Figure 3. The polarization index of the transformer with new oil is 1.49 while the one with aged oil is 4.55. It indicates that the polarization index increases with the aging of transformers which is totally misleading!

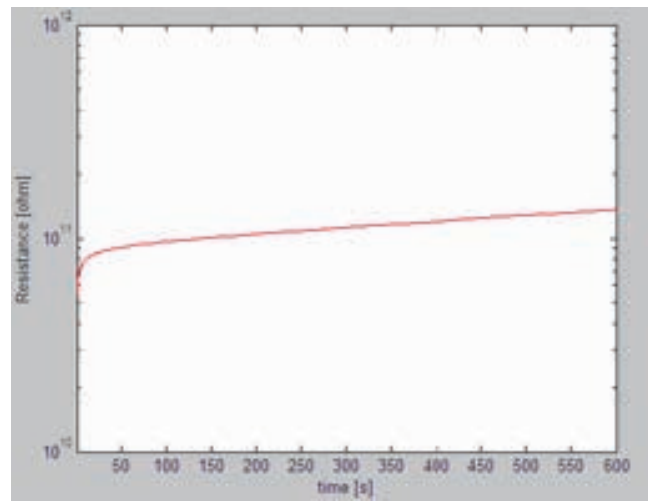

(a)

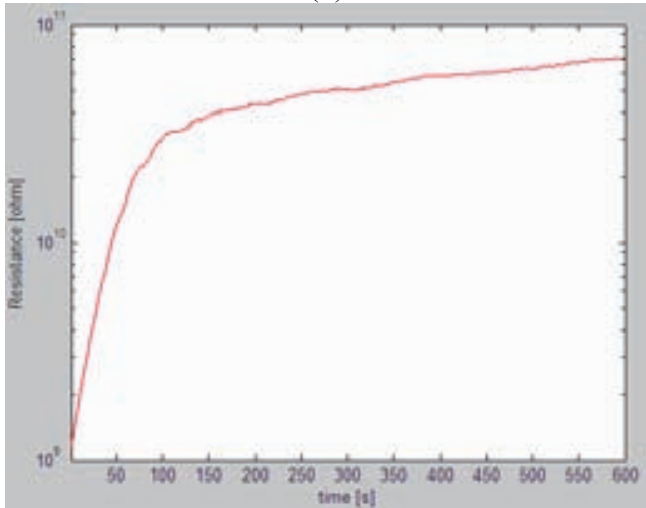

(b)

Figure 3 The measured resistance curve of the power transformer with (a) new oil (b) aged oil

\subsection{Dissipation factor (tan $\delta$ ) test}

The widely accepted and most common insulation diagnostic test is carried out measuring the capacitance and dissipation factor of the insulation at line frequency $(50 / 60 \mathrm{~Hz})$. This test is performed whenever there is a need for insulation property investigation. Analysis is based on the comparison historical values against factory values. Since insulation properties are temperature dependent, temperature compensation has to be used for measurements not performed at $20 \square \mathrm{C}$ which is 
normally achieved using temperature correction table factors for certain classes of devices [4] recommended by the international standards.

However, the dissipation factor of insulation materials in good condition is usually quite small and could be vulnerable to the unexpected surface current leakage path [5] and contaminations [6]. In some extreme cases the dissipation factor shows a negative value. In addition to that, the recommended factors for temperature correction are only average values and therefore subject to error. The classic results presented by Blodget [7] in Figure 4 show the temperature dependent power factor of oil-paper insulation with different moisture contents. The power factor of wet insulation is more temperature dependent than that of the dry insulation. Transformers with different geometry (volume ratio of oil and paper) also have different correction factors.

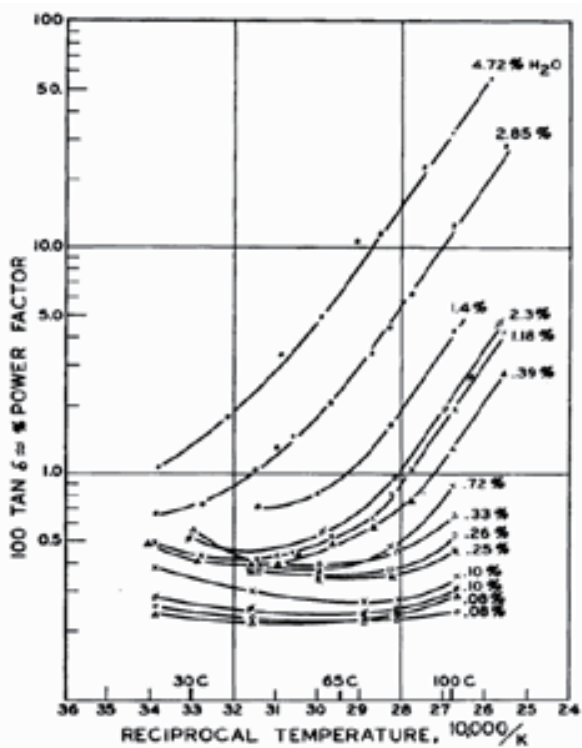

Figure 4 Power factor at $60 \mathrm{~Hz}$ for oil-paper insulation with various moisture contents as a function of temperature $\left({ }^{\circ} \mathrm{C}\right)[7]$

The $50 / 60 \mathrm{~Hz}$ dissipation factors obtained at different temperatures is not a perfect trending method because of the temperature correction limitation.

\subsection{Recovery voltage method}

The recovery voltage method, RVM technique has been, by far, a very popular method for insulation condition assessment. It is an advanced diagnostic method used to estimate the moisture content of the insulation from the voltage relaxation measurement.

However, there has been much controversy surrounding the technique [8]. Moisture determinations are often much higher than obtained by other methods. The reason is that it does not take the influence of oil into account. In other words, it lacks the modeling of the insulation system and its interpretation scheme is simplistic as described in [9].

\section{Review of the Dielectric Frequency Response method}

The Dielectric Frequency Response, DFR method is the extension of $\tan \delta$ measurement at power frequency. The measurement principle and setup is very similar to traditional $50 / 60 \mathrm{~Hz}$ power factor testing with the difference that a lower measurement voltage is used $(200 \mathrm{Vp})$, insulation properties are measured over a frequency range, typically from $1 \mathrm{kHz}$ to $1 \mathrm{mHz}$. In frequency domain each material is characterized by a complex permittivity $\varepsilon_{r}^{*}$ :

$$
\varepsilon_{r}^{*}=\frac{C_{x}}{C_{0}}=\varepsilon_{r}^{\prime}-j \varepsilon_{r}^{\prime \prime}
$$

where $C_{x}$ is the measured capacitance and $C_{0}$ is the geometrical capacitance. The loss tangent can be expressed by:

$$
\tan \delta=\frac{\varepsilon_{r}^{\prime \prime}}{\varepsilon_{r}^{\prime}}
$$

The results are normally presented as capacitance and loss/tan delta/power factor versus frequency such as shown in Figure 5. The curve represents the unique characteristics of the insulation.

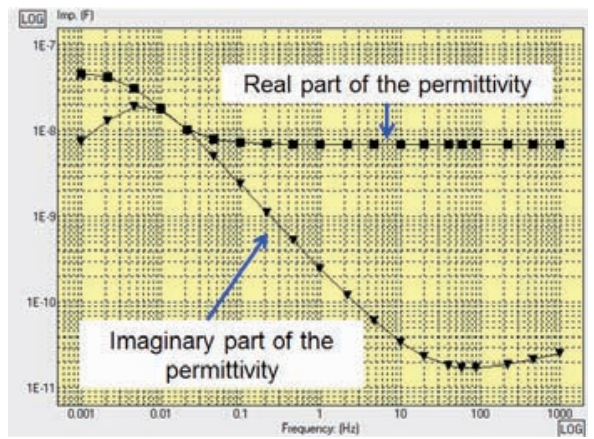

Figure 5 Typical DFR measurement results

The characteristic curve of power transformers at various frequencies $\varepsilon_{\text {trans }}^{*}(\omega)$ is a function of cellulose property $\varepsilon_{c e}^{*}(\omega)$, oil property $\varepsilon_{\text {oil }}^{*}(\omega)$, geometry $\mathrm{G}$ (the way cellulose and oil are combined) and temperature $\mathrm{T}$, which could be written as:

$\varepsilon_{\text {trans }}^{*}(\omega)=\mathbf{F}\left(\varepsilon_{c e}^{*}(\omega), \varepsilon_{\text {oil }}^{*}(\omega), G, \mathrm{~T}\right)$

$\varepsilon_{c e}^{*}$ is the complex permittivity of cellulose which is mainly affected by moisture. Typical 
moisture dependent characteristic curves of paper/cellulose are shown in Figure 6 [10].
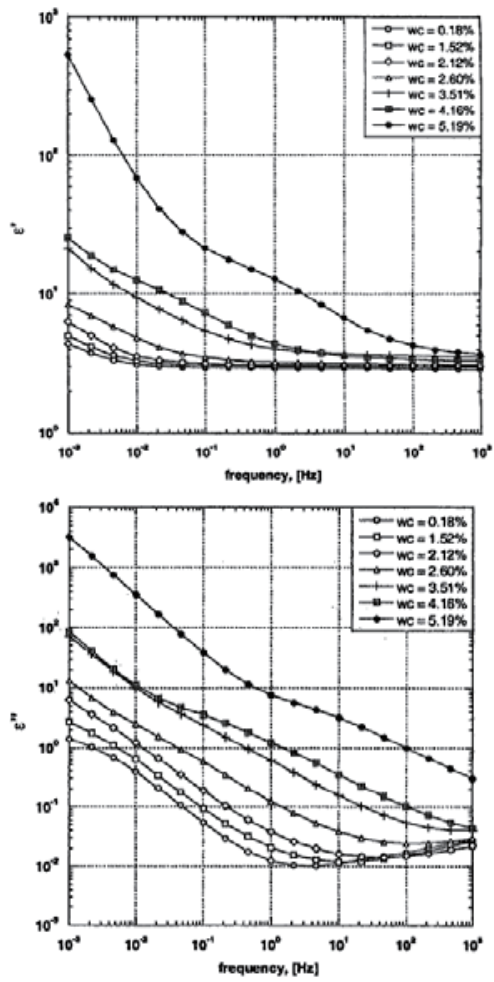

Figure 6 Real and imaginary part of the permittivity of the Kraft paper at different moisture contents [10]

For transformer oil, the real part permittivity is constant $\left(\varepsilon_{r}=2.2\right)$. The imaginary part is dominated by the DC conductivity $(\sigma)$ which could be represented by a straight line in frequency domain as shown in Figure 7 [11].

$$
\varepsilon_{\text {oil }}^{*}(\omega)=2.2-j \frac{\sigma}{\omega \varepsilon_{0}}
$$

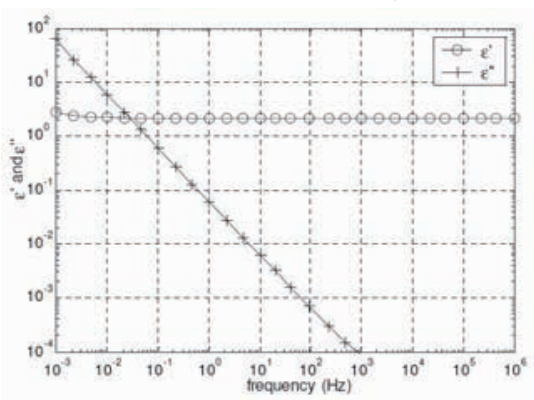

Figure 7 Real and imaginary part of the permittivity of the mineral oil [11]

The geometry of the transformer insulation is used to describe the relative amount of oil and cellulose and how they are combined. The most widely used model is called XY model [12] which is constructed based on the structure of the cooling duct.
Parameter $\mathrm{X}$ is defined as the ratio of the sum of all barriers in the duct, lumped together, and divided by the duct width. The spacer $Y$ is defined as the total width of all the spacers divided by the total length of the periphery of the duct as shown in Figure 8.

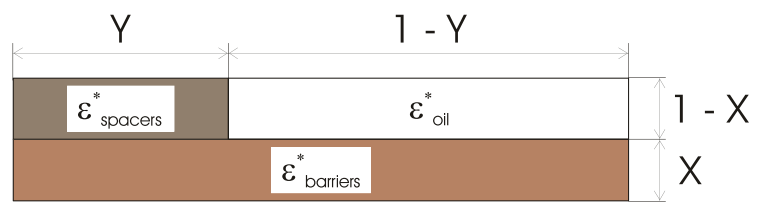

Figure $8 \quad \mathrm{XY}$ model as described in [12]

Knowing the permittivity of cellulose and oil, as well as their amount and the way they are combined, the permittivity of a power transformer is given by:

$$
\begin{aligned}
& \varepsilon_{\text {trans }}^{*}(\omega)=\frac{Y}{\frac{1-X}{\varepsilon_{\text {spacer }}^{*}}+\frac{X}{\varepsilon_{\text {barrier }}^{*}}}+\frac{1-Y}{\frac{1-X}{\varepsilon_{\text {oil }}^{*}}+\frac{X}{\varepsilon_{\text {barrier }}^{*}}} \\
& \text { Where } \quad \varepsilon_{\text {spacer }}^{*} \quad \text { and } \quad \varepsilon_{\text {barrier }}^{*} \text { are the }
\end{aligned}
$$
permittivity of cellulose which could be expressed by $\varepsilon_{c e}^{*}$.

The temperature dependence of cellulose and oil are different. DFR method gives a reasonable and logical mathematical approach regarding the thermal behavior of the system. In general, the increase/decrease of temperature makes the dielectric spectrum shift towards higher/lower frequencies at the logrithmic scale while the shape remains unchanged. Figure 9 is an example of the dielectric frequency response of cellulose at different temperatures. The shifted distance is determined by the activation energy of the material. The activation energy of the Kraft paper is about 1-1.05 eV while mineral oil has activation energy of $0.4-0.5 \mathrm{eV}$ [13]. The higher the activation energy, the further DFR curves shift at a given temperature increase.

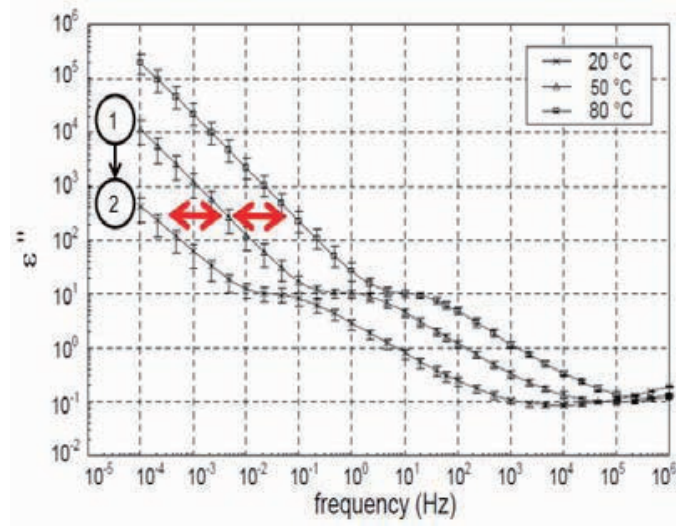

Figure 9 DFR curves of cellulose at different temperatures [11] 
After solving the function containing these parameters, the DFR method gives by far the most accurate results compared with other methods. Figure 10 shows the moisture determination of a oil-paper system using the DFR method (MODS software) at different temperatures compared with the Karl Fisher titration method [14]. It supports the argument that the DFR method could give the reliable moisture determination of insulation systems at different temperatures.

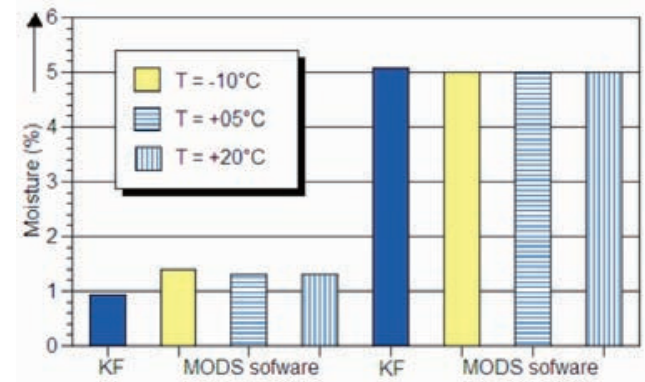

Figure 10 Comparison of DFR analysis with Karl Fisher method for moisture determination in the oil-paper insulation [14]

In practice, the insulation temperature needs to be carefully measured and put into the post-analysis software By varying the remaining three variables in the function $\mathrm{F}$, a best fit to the measured characteristc curve could be obtained. Thus the moisture content of cellulose, oil conductivity/loss tangent, relative amount of cellulose and oil from the database represent the measured transformer as shown in Figure 11.

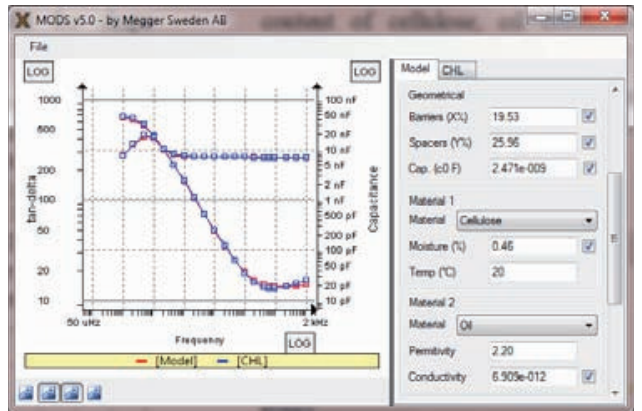

Figure 11 MODS ${ }^{\circledR}$ moisture and oil analysis for a 20 MVA transformer

\section{Latest discoveries}

The most controversial topic is whether $\mathrm{X}$ and $\mathrm{Y}$ should be fixed according to the designed parameters or let vary to get the best fit during post analysis. More detailed transformer model is analyzed by the Finite Element method [15] so that the differences of two methods are known. Fortunately, the matching reuslts are not very sensitive to the insulation geometry. Results obtained by two methods are listed in [15] and it shows that the moisture content of cellulose, oil conductivity and tan delta of the transformer given by different methods just have slight differences with each other.

Power factor/dissipation factor testing is usually performed at $10 \mathrm{kV}$ or the readings are converted to $10 \mathrm{kV}$ equivalent. One reason that industries standardized on a $10 \mathrm{kV}$ test voltage is for immunity against electrostatic interference. Power transformers with oil-paper type insulating systems in good condition exhibit a flat response when power factor/dissipation factor $(50 / 60 \mathrm{~Hz})$ is measured as a function of the test voltage [16].

The DFR method uses a 140V RMS test voltage signal that could be amplified up to $30 \mathrm{kV}$ with an external amplifier. However, the response at lower frequencies shows certain voltage dependence. The DFR curves of a 20 MVA power transformer at various test voltages ranging from $20 \mathrm{~V}$ to $1.4 \mathrm{kV}$ are shown in Figure 12 . The distortion at lower frequencies is because of the non-linear characteristics of the oil-paper system. When these two media are put into contact (forming interfaces) under test voltage, charge accumulation occurs at the interfaces due to the differences between their electrical properties. This kind of polarization is the Maxwell-Wagner [9] effect or interfacial polarization. The effect of the polarization is determined by the field strength formed by the test voltage.
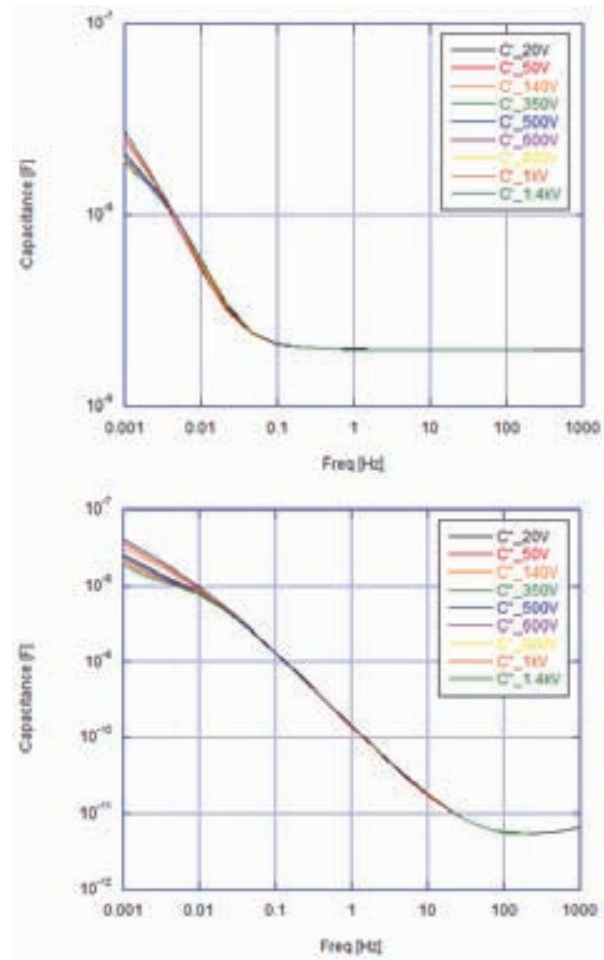

Figure 12 DFR curves of a 20MVA power transformer at different voltages 


\section{Conclusions}

This article reviews the widely used technologies for transformer moisture estimation. Limitations, merits and guidelines for better interpretation are provided throughout the contet of the document..

The DFR method is demonstrated to be an excellent tool for the off-line assessment of power transformer insulation. Moisture content of cellulose, oil conductivity of power transformers could be reliably estimated from the measurement at all temperatures. DFR performance in the field using high voltages shows a deviation at the very low frequencies, this is explained under the Maxwell-Wagner effect.

\section{References}

[1] Yanqing Du, et al., Moisture equilibrium in transformer paper-oil systems, IEEE Electrical Insulation Magazine, vol. 15, no. 1, pp. 11-20, 1999

[2] Peter A. Bruttel, et al., Water determination by Karl Fischer Titration, Metrohm AG

[3] Maik Koch, et al., Reliability and Improvements of Water Titration by the Karl Fischer Technique, 15th ISH 27-31 August 2007 Ljubljana, Slovenia

[4] IEEE Guide for Diagnostic Field Testing of Electric Power Apparatus; Part 1: Oil Filled Power Transformers, Regulators, and Reactors, IEEE 62-1995

[5] Huang Minru, et al., Analysis and Treatment on $\tan \delta$ Measuring Error of Oil Paper Capacitive Bushing, High Voltage Apparatus, vol. 44, no. 5, pp. $483-485,2008$

[6] Jialu Cheng, et al., Improvements of the Transformer Insulation XY Model Including Effect of Contamination,

[7] Blodget R.B., Influence of Absorbed Water and Temperature on Tan Delta and Dielectric Constant of Oil-Impregnated Paper Insulation, Trans. AIEE, 1961

[8] Xose M. Lopez-Fernandez, et al., Transformers: Analysis, Design, and Measurement

[9] Stainslaw M. Gubanski, et al., Dielectric Response Methods for Diagnostics of Power Transformers

[10] Roberts Neimanis, Determination of Moisture Content in Mass Impregnated Cable Insulation Using Low Frequency Dielectric Spectroscopy, IEEE Power Engineering Society Summer Meeting, 2000

[11] Chandima Ekanayake, Diagnosis of Moisture in Transformer Insulation - Application of frequency domain spectroscopy, Chalmers University of Technology, 2006
[12] Uno Gafvert, et al., Dielectric Spectroscopy in Time and Frequency Domain Applied to Diagnostics of Power Transformers, Proc. Of the 6th ICPADM, Xi' an, China, 2000

[13] Dag Linhjell, et al., Dielectric Response of Mineral Oil Impregnated Cellulose and the Impact of Aging, IEEE Transactions on Dielectrics and Electrical Insulation, Volume: 14 , Issue: 1, 2007

[14] Issouf Fofana, et al., Low Temperature and Moisture Effects on Oil-Paper Insulation Dielectric Response in Frequency Domain, IEEE Electrical Insulation Conference, 2009

[15] Ohlen, Matz, et al., Best practices for Dielectric Frequency Response measurements and analysis in real-world substation environment, CMD international conference, 2012

[16] Dinesh Chhajer, Electrical Tester, Megger Limited, 2012

Cheng Jialu received his BS degree in electrical engineering in 2009 from the Southeast University, Nanjing, China, and MS degree in power system engineering in 2011 from Royal Institute of Technology, Sweden. From 2011 to present, he focuses on research and application of power transformers diagnosis in Megger.

Email: Jialu.cheng@megger.com 